【环境与能源 / Environment and Energy】

\title{
悬浮快速过滤工艺处理晴天污水效果初探
}

朱红生 ${ }^{1,2}$, 李 阳 $^{1}$, 杨云安 ${ }^{3}$, 陈 俊 $^{3}$, 千种健理 ${ }^{4}$, 林 野 $^{4}$, 管运涛 ${ }^{1}$

1) 清华大学深圳研究生院，广东深圳 $518055 ； 2 ）$ 清华大学环境学院，国家环境保护环境微生物利用与 安全控制重点实验室，北京 $100084 ； 3 ）$ 常州市排水管理处，江苏常州 $213077 ； 4 ）$ 日立造船株式会社，日本东京 1400013

摘 要: 悬浮快速过滤工艺作为合流雨污水溢流污染处理技术已有应用案例，但该工艺对晴天污水的 处理效果尚未得到确认，在中国江南某市合流区域进行了晴天污水处理的现场中试，结果表明，悬浮快速 过滤工艺对污水的悬浮颗粒物 (suspended solid, SS) 和化学需氧量 (chemical oxygen demand, COD) 具有较高 的去除率. 其中, SS 去除率约为 $29.5 \% \sim 58.3 \%, \mathrm{COD}$ 的去除率约为 $6.7 \% \sim 42.6 \%$ 。高滤速和小预处理 单元水力负荷有利于提高悬浮快速过滤工艺的污染物去除率. 进水水质也影响着处理效果. 该工艺较适用 于高 SS 和高非溶解态 COD 比例的污水，进水中极小粒径颗粒物 $(<25 \mu \mathrm{m})$ 的比例是工艺对 SS 的去除率的 限制因素，而进水中 SS、非溶解性 COD 与极细粒径颗粒物 $(<25 \mu \mathrm{m})$ 比例可作为工艺适应性的关键性指 标. 同时，将合流制溢流的悬浮快速过滤装置作为污水的预处理设施，对减少合流管沉积物具有重要意义. 关键词：环境工程学；悬浮快速过滤；晴天污水处理；合流制溢流；污染物去除率；粒径分布 中图分类号：TU992 文献标志码：A doi：10.3724/SP. J.1249.2020.02187

\section{Preliminary study on the effect of suspension rapid filtration process on sewage treatment in sunny days}

\section{ZHU Hongsheng ${ }^{1,2}$, LI Yang ${ }^{1}$, YANG Yun'an ${ }^{3}$, CHEN Jun ${ }^{3}$, CHIKUSA Takemasa ${ }^{4}$, HAYASHI No ${ }^{4}$, and GUAN Yuntao ${ }^{1}$}

1) Graduate School at Shenzhen, Tsinghua University, Shenzhen 518055, Guangdong Province, P. R. China

2) School of Environment, Tsinghua University, State Environmental Protection Key Laboratory of

Microorganism Application and Risk Control, Beijing 100084, P. R. China

3) Changzhou Drainage Administration, Changzhou 213077, Jiangsu Province, P. R. China

4) Hitachi Zosen Corporation, Tokyo 1400013, Japan

\begin{abstract}
The suspension rapid filtration process has been applied as a pollution treatment technology for combined sewage overflow, but the treatment effect on treating sewage during sunny days has not been confirmed. In this paper, we carry out the on-site pilot test of sunny sewage treatment in a combined sewage area (i. e. a certain city of Jiangnan). The test results indicate that the suspension rapid filtration process effectively removes the suspended solid (SS) and chemical oxygen demand (COD) in sewage, the removal rates of SS and COD are from 29.5\% to $58.3 \%$ and from $6.7 \%$ to $42.6 \%$, respectively. High filtration velocity and small hydraulic load of pretreatment
\end{abstract}

Received: 2018-06-18; Accepted: 2018-11-30

Foundation: National Science and Technology Major Special Project of China (2017ZX07202002) ; Shenzhen Science and Technology Research Foundation (JSGG20160428181710653, JSGG20170412145935322)

Corresponding author: Professor GUAN Yuntao. E-mail: guanyt@ tsinghua.edu.cn

Citation: ZHU Hongsheng, LI Yang, YANG Yun'an, et al. Preliminary study on the effect of suspension rapid filtration process on sewage treatment in sunny days $[\mathrm{J}]$. Journal of Shenzhen University Science and Engineering, 2020, 37(2) : 187-193. (in Chinese) 
unit are beneficial to improve pollutant removal efficiency of pollutants in the suspension rapid filtration process. The influent quality also affects the treatment effect. The process is more applicable to the treatment of sewage with high SS and insoluble COD ratio. The proportion of SS particles $<25 \mu \mathrm{m}$ in influent is also key limiting factors to the removal rate of SS. And, the ratio of SS, insoluble COD and ultra-fine particles $(<25 \mu \mathrm{m})$ in influent can be used as key indicators of process adaptability. Meanwhile, the suspension rapid filtration device can also be applied as a pretreatment facility for sewage to treat the combined overflow, which is of great significance to reduce sediment in combined sewer pipes.

Key words : environmental engineering; suspended rapid filtration; sewage treatment; combined sewer overflow; pollutant removal rate; particle size distribution

传统的合流制排水系统在雨天易发生污水溢 流，因溢流中包含大量的生活污水、管道沉积物和 雨水径流，使得部分污染物浓度甚至远超一般生活 污水, 严重污染了受纳水体 ${ }^{[1-5]}$, 是造成水体黑臭 的重要原因. 为控制合流制溢流对受纳水体的污 染，国家重大水专项项目对中国江南某市老城区区 域的合流制排水系统展开了充分的现场调研和采样 监测研究，针对难以截流和收集的溢流污水开发了 一套悬浮快滤处理工艺. 该工艺利用可悬浮于水中 的滤料, 下部进水、上部出水，使悬浮滤料受到上 向水流力挤压成层后形成滤层 ${ }^{[6-7]}$, 该滤层依靠滤 料受到的浮力形成空隙, 实现对水中悬浮颗粒物的 拦截和黏附，以达到过滤的目的. 相对传统过滤, 悬浮过滤的滤层有截污容量大、水头损失增长缓 慢、总水头损失小、过滤周期长等特点 ${ }^{[6]}$. 相关研 究结果表明, 悬浮快速过滤工艺能够有效地控制溢 流、污水污染，对雨污水中悬浮颗粒物（suspended solid, SS) 及其吸附的有机物去除效果很好 ${ }^{[3,8-10]}$. 李思远 ${ }^{[3]}$ 研究表明, 悬浮快滤池在高滤速下对 SS 的平均去除率可达 $45 \%$ 以上. 王润娟等 ${ }^{[8]}$ 研究表 明，最优试验条件下，上向流悬浮滤料滤池对原水 $\mathrm{SS}$ 、浊度及总磷（total phosphorus，TP）的平均去 除率分别为 $86 \% 、 90 \%$ 和 $64 \%$.

合流制溢流一般发生在降雨期，而非雨期时悬 浮快滤池闲置, 设备利用率较低. 因此, 悬浮快速 过滤工艺处理晴天污水的研究，一方面可以有效提 高装置的利用率、去除污水中悬浮颗粒物以减少管 道沉积, 另一方面可以探究影响工艺处理效果的因 素, 为雨天运行与推广该工艺提供参考. 本研究结 合前期研究成果和经验 ${ }^{[3,9]}$ ，与日本日立造船株式 会社合作，对合流制排水区域的悬浮快速过滤工艺 处理晴天污水的效果进行研究，探究该工艺适用的 处理对象水质范围，分析工艺出现的工程问题并提
供解决方案.

\section{1 材料与方法}

悬浮快速过滤工艺设施设置于江南某市老城区 中合流制排水未改造的城中村排水洜站, 区域面积 约为 $55000 \mathrm{~m}^{2}$, 服务人口约为 2750 人. 本研究以 最大处理规模为 $640 \mathrm{~m}^{3} / \mathrm{d}$ 的中试装置的运行数据 为研究基础. 该悬浮快速过滤装置采用的是烯烃系 列纤维的新型滤料, 其形状为圆柱体, 滤材直径为 $8 \mathrm{~mm}$, 滤材长度为 $8 \mathrm{~mm}$. 运行时污水先进人预处 理单元，然后自下而上进人滤池进行过滤，滤后进 人出水池排出装置. 反冲洗采用曝气反冲洗. 填料及 滤料示意见图1.

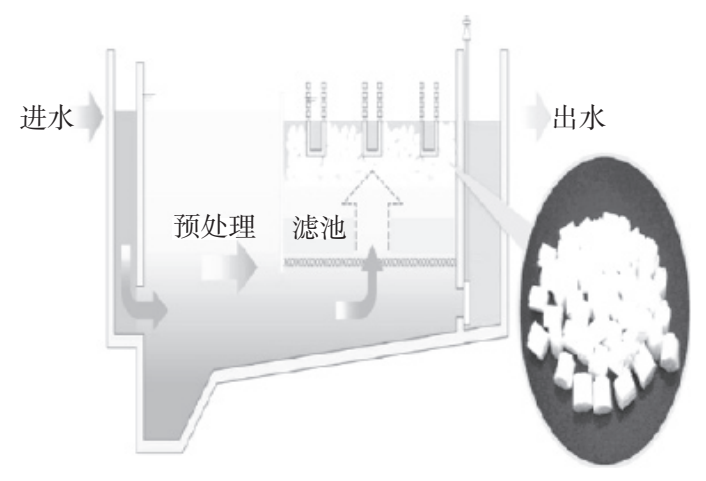

图 1 悬浮快速过滤池和滤料示意图

Fig. 1 Diagrammatic sketch of suspended quick filter tank and filter material

泵站截流区域的污水和雨水进人调蓄池. 雨天 时, 当调蓄池达到一定水位时运行快速过滤工艺, 实现对该区域雨污混合水的快速处理与排出，从而 有效减少该区域的洪涝灾害和溢流污染; 晴天时， 悬浮快速过滤工艺可最大限度地去除污水中的悬浮 颗粒物，以减少管道沉积物. 
对该装置共进行了 6 次晴天运行采样试验, 每 隔 $1 \mathrm{~h}$ 采集进、出水水样, 采样信息见表 1 . 其中, 回送流量是指进人滤池的未过滤的原水回流至预处 理单元的流量, 其目的是在保证滤速相同的情况 下, 适当提高工艺预处理单元的水力负荷.

测定指标包括 SS、化学需氧量 (chemical oxygen demand, COD ) 、溶解性 COD ( soluble COD, $\mathrm{SCOD}) . \mathrm{SS}$ 和 COD 测定方法分别采用重量法、重 铬酸钾快速消解法, SCOD 测定时水样先经过孔径
为 $0.45 \mu \mathrm{m}$ 的滤膜过滤, 然后测定过滤后水样的 COD. 为减少误差, 平行采集几组进水水样进行混 合, 得到进水的混合水样, 采用同样的方法得到出 水的混合水样，然后采用湿法分粒径篮分的方 法 $^{[3]}$, 将进、出水各自的混合样分别依次经过孔径 为 $25 、 45 、 100 、 300 、 1000$ 和 $2000 \mu \mathrm{m}$ 的不锈钢 篮进行篮分，再用蒸馏水进行反冲，测定不同粒径 范围内颗粒物质量.

表 1 六次实验采样信息

Table 1 Sampling information of six sunny experiment

\begin{tabular}{cccccccc}
\hline $\begin{array}{c}\text { 实验 } \\
\text { 组号 }\end{array}$ & $\begin{array}{c}\text { 采样 } \\
\text { 日期 }\end{array}$ & $\begin{array}{c}\text { 采样 } \\
\text { 历时 } / \mathrm{h}\end{array}$ & $\begin{array}{c}\text { 采样 } \\
\text { 间隔 } / \mathrm{h}\end{array}$ & $\begin{array}{c}\text { 进水流量/ } \\
\left(\mathrm{m}^{3} \cdot \mathrm{h}^{-1}\right)\end{array}$ & $\begin{array}{c}\text { 回送流量/ } \\
\left(\mathrm{m}^{3} \cdot \mathrm{h}^{-1}\right)\end{array}$ & $\begin{array}{c}\text { 过滤速度/ } \\
\left(\mathrm{m} \cdot \mathrm{h}^{-1}\right)\end{array}$ & $\begin{array}{c}\text { 预处理单元水力负荷/ } \\
\left(\mathrm{m}^{3} \cdot \mathrm{m}^{-2} \cdot \mathrm{h}^{-1}\right)\end{array}$ \\
\hline $\mathrm{A}$ & $2015-12-23$ & 12 & 1 & 26.67 & 0 & 20.83 & 24.42 \\
$\mathrm{~B}$ & $2015-12-30$ & 10 & 1 & 21.33 & 0 & 16.67 & 19.54 \\
$\mathrm{C}$ & $2016-01-05$ & 15 & 1 & 16.00 & 0 & 12.50 & 14.67 \\
$\mathrm{D}$ & $2015-12-18$ & 6 & 0.5 & 31.85 & 5.18 & 20.83 & 29.17 \\
$\mathrm{E}$ & $2016-01-20$ & 17 & 1 & 31.85 & 10.52 & 16.67 & 29.17 \\
$\mathrm{~F}$ & $2016-01-25$ & 17 & 1 & 31.85 & 15.85 & 12.50 & 29.17 \\
\hline
\end{tabular}

\section{2 结果与讨论}

\section{1 悬浮快速过滤工艺处理污水的影响因素}

\section{1.1 预处理单元水力负荷}

悬浮快速过滤工艺中存在预处理单元，预处理 单元类似污水处理工艺中的配水井，使得进人滤池 的污水趋向稳定, 其水力负荷是滤池处理效果的重 要因素. 为研究预处理单元对悬浮快速过滤工艺处 理效果的影响, 选择 $\mathrm{A}$ 和 $\mathrm{D}$ 组、 $\mathrm{B}$ 和 $\mathrm{E}$ 组以及 $\mathrm{C}$ 和 $\mathrm{F}$ 组为研究对象, 这 3 个对应实验组的两两之间 过滤速度相同，但预处理单元的水面积负荷差异较 大. 图 2 为 $\mathrm{A} 、 \mathrm{~B} 、 \mathrm{C}$ 组的进、出水水质和平均去 除率, 图 3 为 $\mathrm{D} 、 \mathrm{E} 、 \mathrm{~F}$ 组的进、出水水质和平均去 除率. 图 2 表明，A、B、C 组的去除率仍然受到进 水水质的影响， $\mathrm{SS}$ 去除率为 $47.7 \% \sim 58.3 \%$, COD 去除率为 $19.9 \% \sim 42.6 \%$, 说明悬浮快速过 滤技术对污水中的 SS 的去除效果要优于 COD. 由 图 2 和图 3 可知，在 $\mathrm{A}$ 组预处理单元水力负荷低于 D 组的情况下, A 组对污染物的去除率高于 D 组. 而 $\mathrm{B}$ 和 $\mathrm{E}$ 组、 $\mathrm{C}$ 和 $\mathrm{F}$ 组也有类似的结论, 因此, 预 处理单元水力负荷是影响悬浮快速过滤工艺的处理 效果的重要因素.
分析结果表明, 理论上, 相同滤速条件下, 预 处理单元水力负荷越小, 其对污染物的去除效果越 好. 预处理单元类似于配水井, 对进人滤池的污水 进行稳定平衡，提高配水的均匀性. 对滤池而言, 配水均匀性越差, 滤池的进水扰动程度越大, 对滤 池的稳定运行越不利，从而过滤效率降低.

\section{1 .2 滤 速}

滤速（滤池滤速）是影响悬浮快速过滤工艺处 理效果的重要因素. 为分析不同滤速对处理效果的 影响, 选择 $D$ (滤速为 $20.83 \mathrm{~m} / \mathrm{h}$ )、 $\mathrm{E}$ ( 滤速为 $16.67 \mathrm{~m} / \mathrm{h}$ ) 和 $\mathrm{F}$ (滤速为 $12.50 \mathrm{~m} / \mathrm{h}$ ) 组作为研究对 象, 图 3 分别是 $\mathrm{D} 、 \mathrm{E} 、 \mathrm{~F}$ 组的进、出水的 $\mathrm{SS}$ 、 COD 随运行时间的变化. 从图 3 可见，总体而言， 工艺出水水质因进水水质的波动而波动，其 $\mathrm{SS}$ 去 除率为 $29.5 \% \sim 37.5 \%, \mathrm{COD}$ 去除率为 $6.7 \%$ $15.1 \%$ 。悬浮快速过滤对 COD 的去除率要比 SS 的 去除率小, 与污水中的 COD 部分以溶解态存在有 关. 3 组实验数据显示, 各组滤速大小依次为 $\mathrm{D}>$ $\mathrm{E}>\mathrm{F}$ ，工艺对 $\mathrm{SS}$ 和 COD 的去除率为 $\mathrm{D}>\mathrm{E}>\mathrm{F}$, 说 明在一定参数范围内相同条件下，滤速越大，过滤 效果越好.

分析表明,较高滤速条件下,与水接触的悬浮滤 料受到的上向流的推动力更大, 滤料之间的孔隙率 


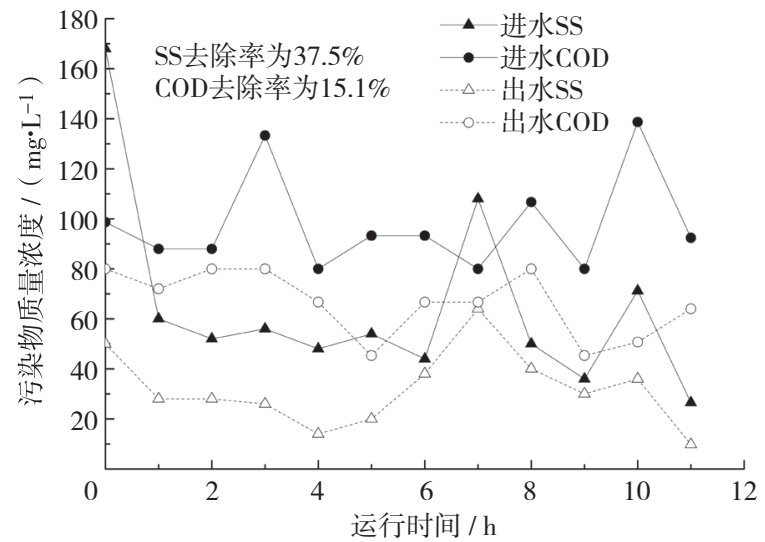

(a) A组

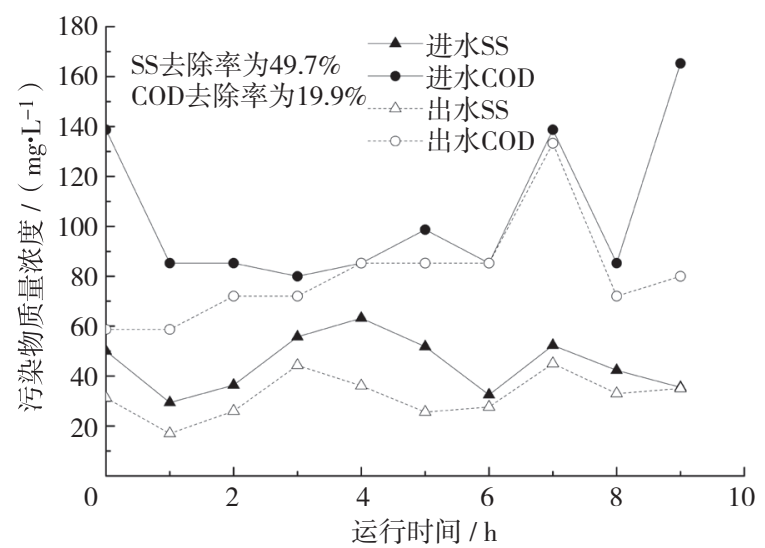

(b) B组

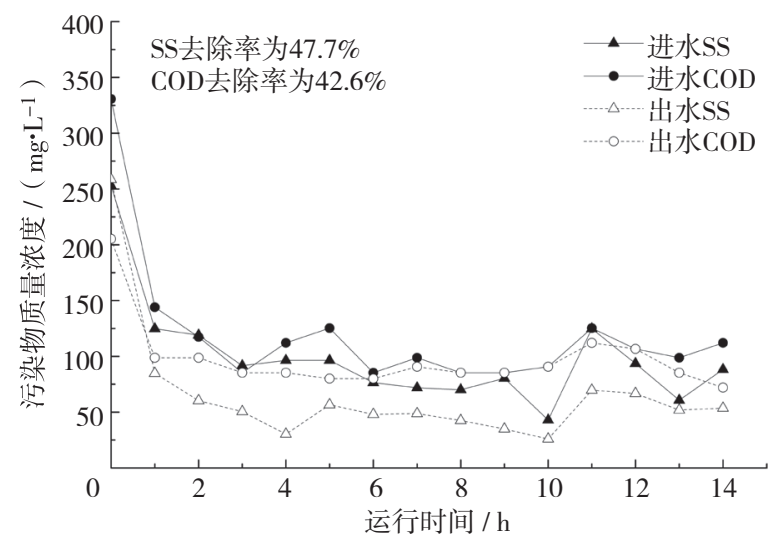

(c) C组

\section{图 $2 \mathrm{~A} 、 \mathrm{~B} 、 \mathrm{C}$ 组的进出水水质和平均去除率}

Fig. 2 Inlet, outlet water quality and average removal rate of group $A, B$ and $C$

更小,从而其对污染物的拦截效果更明显,对污染物 的去除率更高. 对悬浮过滤而言,一定条件下, 滤速 越大,上向水流力挤压成层后形成滤层的密实度越 高, 孔隙越小, 颗粒态拦截和吸附效果越好 ${ }^{[6-7]}$.

\section{1 .3 进水水质}

一般而言，进水水质对反应器的处理效果具有

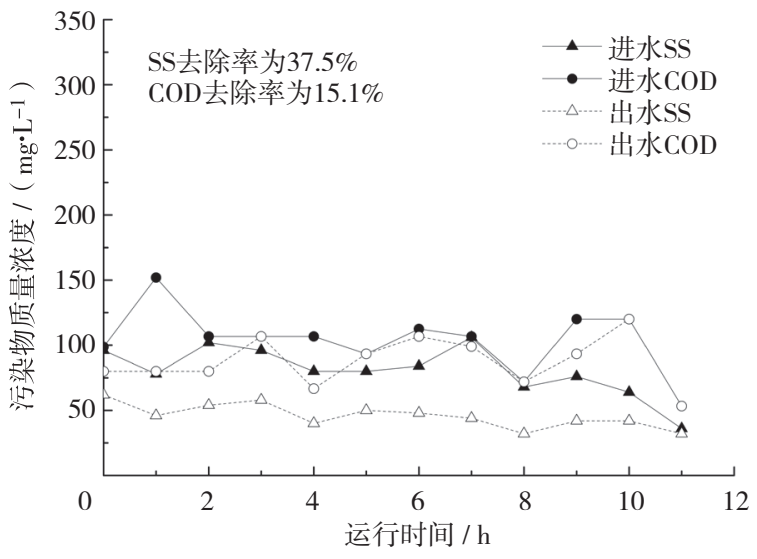

(a) D组

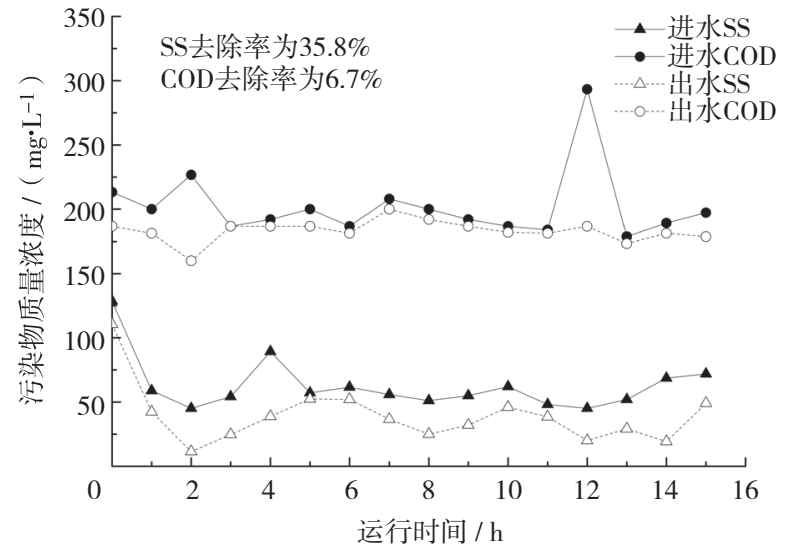

(b) E组

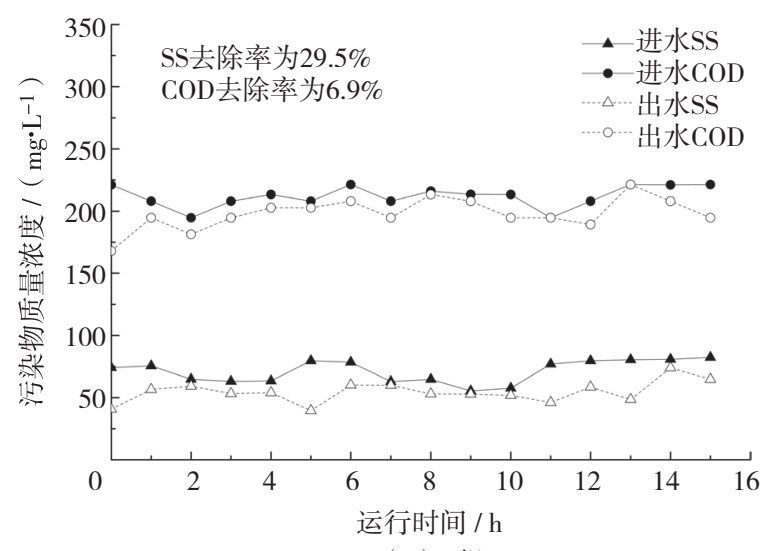

(c) F组

\section{图 $3 D 、 E 、 F$ 组的进出水水质和平均去除率}

Fig. 3 Inlet, outlet water quality and average removal rate of group $D, E$ and $F$

较大的影响. 为探究进水水质对悬浮快速过滤工艺 处理效果的影响, 以进水 $\mathrm{SS}$ 质量浓度为研究对象, 将高于平均质量浓度的 SS 进水统计为高 SS 进水, 低于平均质量浓度的 SS 进水统计为低 $\mathrm{SS}$ 进水, 可 发现高质量浓度 SS 进水平均去除率基本高于低质 量浓度 SS 进水的平均去除率. 因此, 在滤速和预 
处理单元水力负荷相同的条件下, 进水 SS 越高, 悬浮快速过滤工艺对污水中 SS 去除效果越好, 需 要反冲洗的时间可能越长.

以 $\mathrm{B} 、 \mathrm{C}$ 和 $\mathrm{E}$ 组进水的 $\mathrm{COD}$ 作为研究对象 (表 2 ), 研究进水 COD 对处理效果的影响. 图 2、图 3 和表 2 表明, 非溶解性 COD 质量分数和悬浮快速 过滤工艺对 COD 的去除率呈正相关，说明进水中 的非溶解性 COD 质量分数越高, 悬浮快速过滤工 艺对污水中的 COD 去除效果越明显.

表 $2 \mathrm{~B} 、 \mathrm{C}$ 和 $\mathrm{E}$ 组处理前进水 COD 分析

Table 2 Inlet average water quality of group B, C and E

\begin{tabular}{cccc}
\hline 组号 & $\begin{array}{c}\rho(\mathrm{COD}) / \\
\left(\mathrm{mg} \cdot \mathrm{L}^{-1}\right)\end{array}$ & $\begin{array}{c}\rho(\mathrm{SCOD}) / \\
\left(\mathrm{mg} \cdot \mathrm{L}^{-1}\right)\end{array}$ & $\begin{array}{c}w(\text { 非溶解性 } \\
\mathrm{COD}) / \%\end{array}$ \\
\hline $\mathrm{B}$ & 95.0 & 80.0 & 20.0 \\
$\mathrm{C}$ & 172.1 & 140.8 & 43.2 \\
$\mathrm{E}$ & 200.0 & 146.7 & 12.7 \\
\hline
\end{tabular}

总体而言，悬浮快速过滤技术对污水中颗粒物 具有良好的拦截和吸附作用，当污水中的 SS 较高、 非溶解性 COD 质量分数较高时, 悬浮快速过滤工 艺对污水的 SS 和 COD 具有更好的处理效果. SS、 非溶解性 COD 与水体中颗粒物相关, 而颗粒物质 量分数越高, 悬浮快速过滤的效果越好, 该结论与 李思远等 ${ }^{[3,8]}$ 的研究一致.

\section{2 进水中颗粒物不同粒径区间过滤效果分析}

悬浮快速过滤工艺主要去除污水中的颗粒物, 因此, 有必要研究污水中颗粒物的粒径分布, 以及 悬浮快速过滤工艺对不同粒径区间的过滤效果.

对该阶段的污水颗粒物粒径分布（表 3 ）研究 发现, $\mathrm{A} 、 \mathrm{C} 、 \mathrm{E}$ 和 $\mathrm{F}$ 组（因现场原因，未测定 $\mathrm{D}$ 和 $\mathrm{E}$ 组污水中颗粒物粒径）污水中的颗粒物以极小 粒径为主 $(<25 \mu \mathrm{m})$ ，其质量分数基本在 $80 \%$ 以 上，而 $<100 \mu \mathrm{m}$ 粒径的颗粒物质量分数为 $95 \%$ 左 右, 说明污水中较大颗粒物（100 $\mu \mathrm{m}$ 以上）质量 分数较少.

对不同粒径区间过滤效果分析（图 4) 发现, 虽然极小粒径 $(<25 \mu \mathrm{m})$ 在进水中所占质量分数 较高, 但悬浮快速过滤工艺对其处理效果有限, 去 除率约为 $21.2 \% \sim 40.5 \%$; 而 $25 \mu \mathrm{m}$ 以上的颗粒物 所占质量分数虽仅为 $20 \%$ 左右, 但悬浮快速过滤工 艺对 $25 \mu \mathrm{m}$ 以上的颗粒物的平均去除率基本在 50\% 以上. 与对应的 $\mathrm{SS}$ 的平均去除率比较, 极小粒径
颗粒物（<25 $\mu \mathrm{m} ）$ 的去除率基本低于 $\mathrm{SS}$ 平均去 除率，25 $\mu \mathrm{m}$ 以上的颗粒物的平均去除率基本高于 $\mathrm{SS}$ 平均去除率. 同时，由预处理单元水力负荷和滤 池滤速可知， A、 C、 E 和 F 组对 SS 的去除率排序 为 $\mathrm{A}>\mathrm{C}>\mathrm{E}>\mathrm{F}$ ，而对极小粒径颗粒物 $(<25$ $\mu \mathrm{m})$ 则为 $\mathrm{F}>\mathrm{E}>\mathrm{C}>\mathrm{A}$ ，极小粒径颗粒物 $(<25$ $\mu \mathrm{m})$ 质量分数和 SS 去除率呈负相关.

表 3 A、C、E、F 组污水中各粒径质量分数分布

Table 3 Particle size distribution proportions

in sunny sewage of group A, C, E and F $\%$

\begin{tabular}{ccccc}
\hline 粒径 $/ \mu \mathrm{m}$ & $\mathrm{A}$ 组 & $\mathrm{C}$ 组 & $\mathrm{E}$ 组 & $\mathrm{F}$ 组 \\
\hline$>2000$ & 0.45 & 1.62 & 0.48 & 0.30 \\
$1000 \sim 2000$ & 0.55 & 0.60 & 1.12 & 0.67 \\
$300 \sim 1000$ & 0.30 & 0.39 & 0.42 & 0.70 \\
$100 \sim 300$ & 1.21 & 3.00 & 1.18 & 1.42 \\
$45 \sim 100$ & 4.59 & 7.75 & 2.10 & 0.85 \\
$25 \sim 45$ & 9.48 & 1.25 & 5.89 & 2.82 \\
$<25$ & 83.41 & 85.40 & 88.81 & 93.24 \\
\hline
\end{tabular}

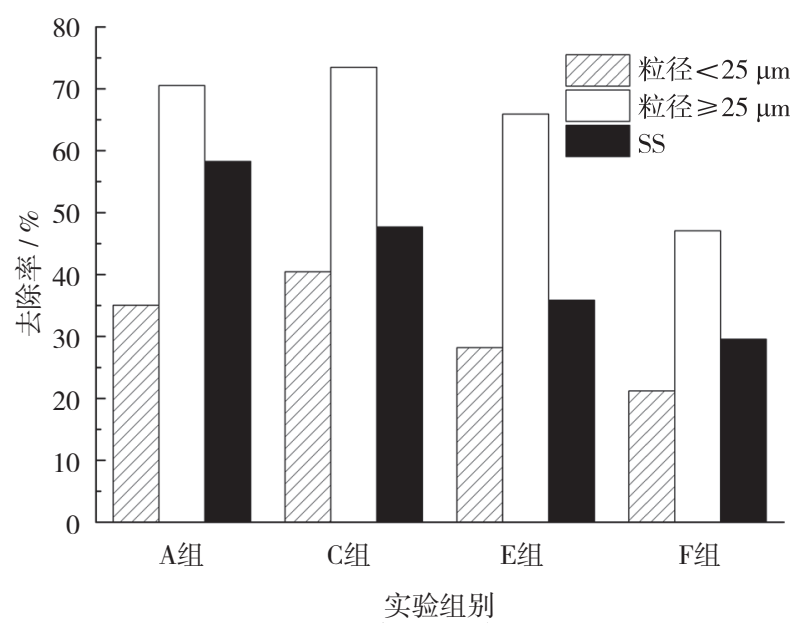

图 4 A、C、E 和 F 组的不同粒径区间颗粒物去除效果

Fig. 4 Removal efficiency in different size range of particle of group A, C, E and F

因此，可认为极小粒径颗粒物（粒径 $<25$ $\mu \mathrm{m} ）$ 是悬浮快速过滤工艺处理效果的限制因素， 污水颗粒物中极小粒径颗粒物（粒径 $<25 \mu \mathrm{m}$ ) 的 质量分数越高, 其处理效果越差. 悬浮过滤时, 上 向水流力压缩形成的滤饼，利用滤饼之间的孔隙对 颗粒物进行拦截和吸附 ${ }^{[6]}$, 颗粒物粒径越小, 拦截 效果越差. 


\section{3 工艺适宜性分析}

悬浮快速过滤工艺对晴天污水的处理效果为: $\mathrm{SS}$ 去除率为 $29.5 \% \sim 58.3 \%$ 、COD 去除率为 $6.7 \% \sim 42.6 \%$ ，低于工艺对合流制溢流 (combined sewer overflow，CSO) 污染物的去除率. 2013 年和 2014 年对某市数个监测点的晴天污水、雨天溢流的 采样监测结果 ${ }^{[3,11-12]}$ 表明, 合流制溢流的 COD 和 SS 质量浓度远高于晴天污水的 (表 4).

表 4 晴天污水与 CSO 污染物平均质量浓度

Table 4 Pollutant average concentrations of sunny wastewater and CSO $\mathrm{mg} / \mathrm{L}$

\begin{tabular}{cccccc}
\hline 样品 & 类别 & $\rho(\mathrm{COD})$ & $\rho(\mathrm{TP})$ & $\rho\left(\mathrm{NH}_{3}-\mathrm{N}\right)$ & $\rho(\mathrm{SS})$ \\
\hline & 春季 & 77.25 & 3.30 & 25.60 & 41.66 \\
& 夏季 & 107.81 & 2.70 & 19.21 & 76.91 \\
晴天 & 秋季 & 88.92 & 1.07 & 20.06 & 62.63 \\
污水 & 冬季 & 66.98 & 3.41 & 11.87 & 45.60 \\
& 平均值 & 85.24 & 2.62 & 19.19 & 56.70 \\
\hline & 最小值 & 33.44 & 0.18 & 3.10 & 22.69 \\
$\mathrm{CSO}$ & 最大值 & 648.84 & 2.09 & 22.22 & 365.61 \\
& 平均值 & 248.28 & 0.97 & 7.28 & 115.40 \\
\hline
\end{tabular}

颗粒物粒径分布是影响工艺处理效果的重要因 素. 表 5 为晴天污水与 $\mathrm{CSO}$ 的颗粒粒径性质比较. CSO 的颗粒物在区间分布上与晴天污水相似，但 $\mathrm{CSO}$ 中颗粒中位粒径与峰值粒径高于晴天污水, 晴 天污水流速较小，能冲刷起的颗粒粒径有限，且污 水中原有大颗粒物质更易沉降，因此晴天污水中颗 粒粒径要小于 CSO.

\section{表 5 晴天污水与 CSO 颗粒粒径分布特性 \\ Table 5 Particle sizes of sunny wastewater and CSO} $\mu \mathrm{m}$

\begin{tabular}{cccc}
\hline 样品 & 粒径集中区间 & 中位粒径 & 峰值粒径 \\
\hline 晴天污水 & $10 \sim 72$ & 19.61 & 23 \\
CSO & $10 \sim 72$ & 22.34 & 52 \\
\hline
\end{tabular}

晴天污水的 SS、COD 和颗粒物粒径均小于 CSO, 导致悬浮快速过滤工艺对晴天污水污染物的 去除率相对较低. 结合前述研究表明，该工艺对特 定水质的污水的处理效果相对较差, 尤其是对低质 量浓度 SS、低质量浓度非溶解性 COD 与高质量浓 度极细粒径颗粒物（粒径 $<25 \mu \mathrm{m}$ ) 的进水而言. 进水中 $\mathrm{SS}$ 、非溶解性 COD 与极细粒径颗粒物（粒 径 $<25 \mu \mathrm{m})$ 的质量浓度可作为悬浮快速过滤工艺
适宜性的关键性指标.

\section{3 结 论}

通过研究在中国江南某市合流制排水区域运用 的悬浮快速过滤工艺对晴天污水的处理效果，结果 表明:

1）悬浮快速过滤工艺对晴天污水的处理效果 为: $\mathrm{SS}$ 去除率为 $29.5 \% \sim 58.3 \%$ 、 $\mathrm{COD}$ 去除率为 $6.7 \% \sim 42.6 \%$. 滤速、预处理单元水力负荷、进 水水质是影响处理效果的主要因素. 理论上, 在一 定适用范围内, 滤速越大, 预处理单元水力负荷越 小, 污水中 $\mathrm{SS}$ 和非溶解性 COD 比例越大, 悬浮快 速过滤技术对 SS 和 COD 的去除效果较好. 悬浮快 速过滤对粒径 $\geqslant 25 \mu \mathrm{m}$ 的颗粒具有较好的去除效 果，极小粒径颗粒物（粒径 $<25 \mu \mathrm{m}$ ) 是影响处理 效果的限制因素.

2）悬浮快速过滤工艺对晴天污水中污染物的 去除率相对 CSO 低，与晴天污水中 $\mathrm{SS} 、 \mathrm{COD}$ 、颗 粒物粒径均小于 CSO 有关. 悬浮快速过滤工艺对低 质量浓度 $\mathrm{SS}$ 、低质量浓度非溶解性 COD 和高质量 浓度极细粒径颗粒物（粒径 $<25 \mu \mathrm{m}$ ) 的污水的处 理效果相对较差. 进水 $\mathrm{SS}$ 、非溶解性 COD 与极细 粒径颗粒物（粒径 $<25 \mu \mathrm{m}$ ) 质量浓度可作为悬浮 快速过滤工艺适用性的关键性指标，其对于推广悬 浮快速过滤工艺的应用具有指导意义.

$3 ）$ 应用于处理合流制溢流的悬浮快速过滤装 置，为提高装置使用率，建议将其作为晴天污水的 预处理设施，以去除污水中悬浮颗粒物，减少管道 沉积物，对控制合流制溢流污染具有重要意义.

\footnotetext{
基金项目：国家科技重大专项资助项目（2017ZX07202002）; 深圳 市科技计划资助项目 (JSGG20160428181710653, JSGG20170412145935322)

作者简介: 朱红生 $(1990-)$, 男, 清华大学硕士研究生. 研究方 向：排水管道污染物控制.E-mail：zhuhs0526@163.com

引文：朱红生,李阳,杨云安, 等. 悬浮快速过滤工艺处理晴 天污水效果初探 $[\mathrm{J}]$. 深圳大学学报理工版, 2020, 36 (2) : 187-193
}

\section{参考文献 / References:}

[1 ] 程 熙，车 伍，唐 否，等. 美国合流制溢流控制 规划及其发展历程剖析 $[\mathrm{J}]$ 。 中国给水排水，2017，33 (6) : 7-12.

CHENG Xi, CHE Wu, TANG Lei, et al. Briefing of the overflow control plan and its development of the combined 
sewer system in the united states $[\mathbf{J}]$. China Water \& Wastewater, 2017, 33(6): 7-12. (in Chinese)

[2] 孙樱珊. 基于 Mike urban 模型的北京市老城区合流制 溢流污染控制研究 $[D]$. 北京: 北京交通大学, 2018 . SUN Yinshan. Study on the pollution control of combined sewer overflow for the old urban in Beijing based on Mike urban model [D]. Beijing: Beijing Jiaotong University, 2018. (in Chinese)

[ 3 ] 李思远. 合流制管网污水溢流污染特征及其控制技术 研究 [D]. 北京: 清华大学, 2015.

LI Siyuan. research on the pollution property of combined sewer overflows and its control technology [D]. Beijing: Tsinghua University, 2015. (in Chinese)

[4] 唐 否, 车 伍, 赵 杨, 等. 合流制溢流初期冲刷 及其控制策略研究 $[\mathrm{J}]$. 给水排水, $2014,40(5)$ ：2430 .

TANG Lei, CHE Wu, ZHAO Yang, et al. Study on initial flushing and control strategy of combined flow overflow [J]. Water \& Wastewater Engineering, 2014, 40 (5) : 24-30. (in Chinese)

[5]李海燕, 徐尚玲, 马 玲. 合流制排水管道沉积物的 研究进展 $[\mathrm{J}]$. 安全与环境学报, 2013, 13(6)：9095.

LI Haiyan, XU Shangling, MA Ling. Progress and prospects of sediments research in the combined sewers $[\mathrm{J}]$. Journal of Safety and Environment, 2013, 13 (6) : 9095. (in Chinese)

[6] 巨志剑. 悬浮滤料过滤技术用于城市污水深度处理的 实验研究 $[\mathrm{D}]$. 西安：西安建筑科技大学, 2005 .

JU Zhijian. Study on filtration with floating media in wastewater advanced treatment [ D ]. Xi'an: Xi'an University of Architecture and Technology, 2005. (in Chinese)

[ 7 ] 田智勇, 何志军, 刘俊良, 等. FILTRAZUR 新型上向 流过滤工艺处理城市地表水 $[\mathrm{J}]$. 给水排水, 2004, 30 (9) : 47-49.

TIAN Zhiyong, HE Zhijun, LIU Junliang, et al. FILTRAZUR: a new process of upward flow filtration treating surface raw water for urban waterworks $[\mathrm{J}]$. Water \& Wastewater Engineering, 2004, 30(9) : 47-49. (in Chi- nese)

[8] 王润娟, 黄 宇, 陈 阳, 等. 上向流悬浮滤料滤池 过滤特性研究及中试应用 $[\mathrm{J}]$. 给水排水, 2016(增 刊): 144-146.

WANG Runjuan, HUANG Yu, CHEN Yang, et al. Filtration characteristics and pilot application of upflow suspension filter media $[\mathrm{J}]$. Water \& Wastewater Engineering, 2016(S1) : 144-146. (in Chinese)

[9]井手幹, 佐大地, 健千種. 簡易型㵶維乃過施設によ る合流改善の実例 $[\mathrm{C}] / /$ 第50 回下水道研究発表会 講演集. [S. l. : s. n. ], 2013, 50.

STEM I, EARTH S, CHIKA K. An example of confluence improvement by simplified fiber filtration facility $[\mathrm{C}] / /$ Proceedings of the 50th Sewerage Research Presentation. Sewerage Research Presentation Lecture Collection. [S. l. : s. n. ] 2013, 50. (in Japanese)

[10] 傅金祥, 唐脪晨, 于鹏飞, 等. 新型纤维滤料高速过 滤污水厂尾水之堵塞及再生 $[\mathrm{J}]$. 中国给水排水, 2017, 33(19) : 33-36.

FU Jinxiang, TANG Yuchen, YU Pengfei, et al. Clogging and regeneration of new fiber filter for treatment of tail waterfrom a WWTP under high speed $[\mathrm{J}]$. China Water \& Wastewater, 2017, 33(19): 33-36. ( in Chinese)

[11] 李思远, 管运涛, 陈 俊, 等. 苏南地区合流制管网 溢流污水水质特征分析 $[\mathrm{J}]$. 给水排水, 2015 (增刊 1 ) : 344-348.

LI Saiyuan, GUAN Yuntao, CHEN Jun, et al. Water quality characteristics of combined sewer overflows in southern region of China $[\mathrm{J}]$. Water \& Wastewater Engineering, 2015(S1): 344-348. (in Chinese)

[12]周美成, 陈 俊, 董良飞, 等. 苏南老城区合流制管 网的溢流污染特征 $[\mathrm{J}]$. 环境工程学报, 2015, 9(7): 3159-3164.

ZHOU Meicheng, CHEN Jun, DONG Liangfei, et al. Pollution characteristics of combined sewer overflows ( CSOs) in old urban area in South Jiangsu $[\mathrm{J}]$. Chinese Journal of Environmental Engineering, 2015, 9 ( 7 ): 3159-3164. (in Chinese)

【中文责编：晨 兮; 英文责编：新 谷】 